\title{
Philosophiques
}

\section{Contenu et objet du jugement chez Brentano}

\section{Guillaume Fréchette}

Volume 38, numéro 1, printemps 2011

Les conférences Hugues Leblanc 2010

URI : https://id.erudit.org/iderudit/1005725ar

DOI : https://doi.org/10.7202/1005725ar

Aller au sommaire du numéro

\section{Éditeur(s)}

Société de philosophie du Québec

ISSN

0316-2923 (imprimé)

1492-1391 (numérique)

Découvrir la revue

Citer cet article

Fréchette, G. (2011). Contenu et objet du jugement chez Brentano. Philosophiques, 38(1), 241-261. https://doi.org/10.7202/1005725ar

\section{Résumé de l'article}

Un des traits dominants de la philosophie autrichienne allant de Bolzano à Husserl est sans nul doute le réalisme logique qui caractérise plusieurs des oeuvres majeures de ce courant de la philosophie. Si ce constat est juste, comme nous le pensons, il tend à masquer le fait que certains concepts clés de ce réalisme logique trouvent leur origine et leur justification dans des théories qui rejettent ces principes réalistes. Ce fait peut être illustré en partant de l'origine du concept d'état de choses dans la philosophie autrichienne, et plus précisément dans la conception des contenus de jugement défendue par Franz Brentano. En exposant les motivations qui le poussent à introduire ces contenus et en analysant les justifications présentées, nous visons à nuancer le constat de départ en illustrant, à l'aide de l'exemple des états de choses, comment les interrelations entre des positions réalistes et nominalistes ont façonné le développement de la philosophie autrichienne. 


\title{
Contenu et objet du jugement chez Brentano
}

\author{
GUILLAUME FRÉCHETTE \\ Université du Québec à Montréal
}

\begin{abstract}
RÉSUMÉ. - Un des traits dominants de la philosophie autrichienne allant de Bolzano à Husserl est sans nul doute le réalisme logique qui caractérise plusieurs des œuvres majeures de ce courant de la philosophie. Si ce constat est juste, comme nous le pensons, il tend à masquer le fait que certains concepts clés de ce réalisme logique trouvent leur origine et leur justification dans des théories qui rejettent ces principes réalistes. Ce fait peut être illustré en partant de l'origine du concept d'état de choses dans la philosophie autrichienne, et plus précisément dans la conception des contenus de jugement défendue par Franz Brentano. En exposant les motivations qui le poussent à introduire ces contenus et en analysant les justifications présentées, nous visons à nuancer le constat de départ en illustrant, à l'aide de l'exemple des états de choses, comment les interrelations entre des positions réalistes et nominalistes ont façonné le développement de la philosophie autrichienne.
\end{abstract}

\begin{abstract}
Logical realism is undoubtedly one of the central features which characterize many of the major works in Austrian philosophy from Bolzano to Husserl. Although this remark is true, as we believe, one must not forget the fact that some of the key concepts of Austrian philosophy are rooted in theories that reject realist principles. As an example, take the concept of state of affairs in Austrian philosophy, and more specifically, Franz Brentano's conception of judgement contents. By showing the motives which led Brentano to introduce these judgement contents and by analyzing the arguments given to support his thesis, the present article aims to contrast the initial remark by illustrating, by means of the case of state of affairs, how the interrelations between realist and nominalist positions have shaped the development of Austrian philosophy.
\end{abstract}

\section{Le réalisme de la philosophie autrichienne et la distinction contenu/objet}

De manière proéminente, les travaux de Kevin Mulligan ont souligné l'importance de l'école de Brentano dans le développement de la philosophie autrichienne et ils ont contribué de manière décisive au renouveau actuel des recherches sur ce courant de la philosophie. Des projets comme celui de l'édition des écrits complets de Brentano, actuellement en cours chez Ontos, ou même l'actualité d'un philosophe comme Bolzano n'auraient certainement pas l'importance qu'ils ont aujourd'hui sans ses nombreuses contributions des trente dernières années ${ }^{1}$.

Un des traits dominants de la philosophie autrichienne de Bolzano à Meinong qu'a bien fait ressortir Mulligan est la tendance réaliste qui y a été

1. Cf. la collection Franz Brentano. Sämtliche veröffentliche Schriften, dont Brentano (2008) et (2009) sont déjà parus. 
développée ${ }^{2}$. En effet, les Sätze an sich de Bolzano comme porteurs de vérité, les Objektive de Meinong, les Bedeutungen et Sachverhalte de Husserl sont autant de spécialités autrichiennes en ontologie et en théorie de la connaissance. Mais ce trait dominant n'est nullement le seul à caractériser les convictions des philosophes autrichiens. Brentano, Marty et plusieurs autres philosophes issus de l'école de Prague défendent des positions difficilement associables au réalisme ${ }^{3}$. En ce sens, on doit distinguer au moins deux grands courants au sein de la philosophie autrichienne, un courant réaliste et un courant nominaliste, comme Mulligan l'a également fait remarquer ${ }^{4}$.

Toutefois, il serait trompeur de voir ces deux courants comme deux positions philosophiques bien ancrées dans des présupposés inquestionnés, soutenus par des philosophes dogmatiques. Brentano lui-même oscille très souvent entre ces deux positions, par exemple. Il me semble plutôt que, dans plusieurs cas, les positions développées par certains philosophes autrichiens sont issues d'une fréquentation de ces deux courants. À mon avis, l'origine autrichienne (ou austro-allemande) du concept d'état de choses ainsi que ses différentes applications en donne justement un bon exemple, et ce tout particulièrement dans le cas de Brentano. Une partie du présent article donnera quelques raisons en faveur de cette compréhension de l'origine à la fois réaliste et nominaliste du concept d'état de choses dans la philosophie autrichienne.

Toutefois, admettre cette double origine ne fournit pas en soi une explication de la variété des positions défendues par Brentano et ses étudiants quant à la nature ontologique des états de choses. Là où Meinong et Husserl admettent les états de choses comme des constituants du monde de plein droit, au même titre que les objets, Brentano et Marty refusent d'en faire une catégorie distincte des contenus de pensée immanents à celui qui juge. C'est pourquoi ils préfèrent normalement parler de contenus de jugements 5 .

Ce que j'aimerais suggérer ici, c'est qu'il y a un moyen efficace de thématiser cette opposition et qui consiste à partir de l'acceptation ou non de la distinction entre le contenu et l'objet des représentations. On peut docu-

2. À titre indicatif, notons seulement Mulligan (1985), (1988), (1993), (1999), (2000), (2001) et Mulligan, Simons \& Smith (1984).

3. L'école de Prague est cette branche pragoise de l'école de Brentano dont le développement est redevable dans une large mesure à Marty. Cf. Fisette et Fréchette (2007), p. 46sq. pour un aperçu général. Parmi les travaux principaux illustrant l'orientation de cette école, $c f$. Kraus (1937), Kastil (1909); (1951), Utitz (1911), Bergmann (1908) et Hillebrand (1891).

4. Cf. Mulligan (1997), p. 122; (1997a), p. 82.

5. Cf. Brentano (1956), p. 38sq. et surtout Marty (1908), p. 228sq. En ce qui a trait à Brentano, bien que l'expression "contenu de jugement» (Urteilsinhalt, ou Inhalt des Urteils) n'intervienne pas comme telle dans la Psychologie d'un point de vue empirique de 1874, elle intervient au plus tard dans les cours de logique de 1875 comme on peut le voir notamment dans EL 81, p. 13608). 
menter cette opposition sur une base historique, comme je le ferai brièvement dans la première section de cet article. Toutefois, les échanges polémiques publiés par les défenseurs de ces positions respectives ne sont pas toujours la meilleure façon de s'orienter dans le débat, car l'idéologie y prend parfois le dessus sur les positions philosophiques discutées et rend ainsi difficile une reconstruction claire des arguments du débat ${ }^{6}$. C'est pourquoi j'opposerai ici les arguments de Twardowski à la position de Brentano (section 3).

En partant de la distinction ontologique entre contenu et objet des représentations, et surtout en examinant pourquoi Brentano ne reconnaît pas une telle distinction, on sera également en mesure de voir ce qui motive son refus de reconnaitre aux états de choses un statut autre que celui de contenu psychologique immanent à l'agent. Ce sera l'objet de la deuxième partie de cet article.

Il peut sembler aujourd'hui inapproprié d'employer l'expression «état de choses» pour désigner un contenu psychologique. C'est que, jusqu'à Husserl, l'expression elle-même est équivoque: par "état de choses" (mais aussi «contenu de jugement» (Urteilsinhalt), «formation" ${ }^{7}$ (Gebilde), " produit $^{8} »($ Produkt), etc.) les philosophes autrichiens pré-husserliens ont rarement été clairs quant à savoir si ces expressions désignaient la signification des jugements, ou si plutôt elles désignaient un objet spécifique au jugement ${ }^{9}$. Le refus des états de choses par Brentano est donc lui aussi équivoque, car il peut aussi bien signifier un refus des états de choses qua signification du jugement (ou de l'énoncé d'un jugement) qu'un refus de ceux-ci qua objets spécifiques du jugement. La plupart des commentateurs s'accordent à dire qu'il refuse la deuxième catégorie, mais certains soutiennent qu'il accepte souvent la première ${ }^{10}$. À mon avis, il refuse les deux. Les états de choses (ou pour parler avec Brentano: les contenus de jugement) sont pour lui des entités mentales inséparables de l'acte dont ils sont le contenu, de manière similaire aux contenus de représentation. Ils sont immanents à

6. C’est le cas notamment chez Marty (1908), p. 388sq, Kastil (1909), p. 180sq. et Kraus (1924). Voir aussi la réponse de Meinong aux représentants de l'école de Prague et surtout à Marty, dans Meinong (1910), p. 97sq.

7. Cf. Stumpf (1907); (2006).

8. Cf. Twardowski (1996); (2007).

9. Un élève de Husserl, Adolf Reinach, remarquait d'ailleurs que «Stumpf, Bergmann, Bolzano - tous les Autrichiens confondent constamment proposition et état de choses ", $c f$. Reinach (1989), p. 526. Husserl lui-même n'est pas toujours parfaitement clair sur cette distinction (cf. Husserl (1900), p. 233-234).

10. Chrudzimski (2001), p. 62sq. et p. 83 ; (2001a), p. 175sq. avance même qu'il reconnaît les deux catégories: des contenus de jugement propositionnels et des états de choses. Rojszczak et Smith (2003), p. 238, ainsi que Chrudzimski et Smith (2004), p. 209, reprennent également cette idée qu'on retrouve sous une autre forme déjà dans Mayer-Hillebrand (1959), p. 320 , où plusieurs des thèses centrales de la phénoménologie husserlienne sont attribuées au jeune Brentano. 


\section{Philosophiques / Printemps 2011}

l'acte et non réels, et ne peuvent donc, par définition, jouer le rôle que jouent les significations chez Husserl ou le sens chez Frege. L'objet du jugement étant pour Brentano normalement celui de la représentation à sa base, celuici ne peut donc être un état de choses au sens d'une entité constituante du monde.

Ce que je suggère ici, c'est que la position défendue par Brentano sur la nature des contenus et des objets de jugement est généralement cohérente de la position qu'il défend sur la non-distinction des contenus et des objets de représentation. En mettant en relief les motivations qui le poussent à maintenir cette non-distinction, on sera donc en mesure de voir comment il peut faire l'économie des deux catégories évoquées plus haut. Certes, sa théorie est confrontée à des difficultés importantes, sur le plan ontologique, et ce particulièrement dans le traitement des jugements existentiels négatifs, mais la reconnaissance de ces catégories ne peut les résoudre.

\section{2. États de choses et contenus de jugements}

Les raisons qui expliquent l'équivocité de l'expression «état de choses» dans la philosophie autrichienne pré-husserlienne peuvent se résumer en gros à l'absence d'un consensus sur la nature du jugement. Pour Bolzano, les énoncés communiquent (kundgeben) des jugements qui ont pour matière (Stoff) des propositions en soi. Contrairement à Brentano, Bolzano ne fait pas de distinction entre l'acte et le contenu du jugement: ce qu'il appelle la matière du jugement, la proposition en soi (Satz an sich), est une entité distincte du jugement et ontologiquement indépendante de celui-ci. L'indépendance ontologique et la fonction sémantique des propositions - les propositions en soi constituent le sens (Sinn) des énoncés - font de celles-ci des candidats tout indiqués pour la première catégorie mentionnée plus haut, celle des états de choses qua significations des jugements.

Pour Brentano, on doit distinguer entre l'acte et le contenu du jugement. Le contenu (Inhalt) de Brentano n'est pas la matière (Stoff) de Bolzano $^{11}$ : pour Brentano, le contenu d'un jugement est ontologiquement dépendant de l'acte (acte et contenu sont mutuellement inséparables ${ }^{12}$ ). Comme nous venons de le voir, ce n'est pas le cas de la matière de Bolzano.

En l'absence d'un consensus, certains successeurs de Bolzano et de Brentano comprennent donc les états de choses comme des contenus de jugement qui jouissent ou non d'une indépendance ontologique face aux actes de jugement ${ }^{13}$. D'autres conçoivent états de choses et contenus de jugement comme des catégories distinctes toutes deux indépendantes ontologi-

11. Malgré ce que plusieurs Autrichiens (et brentaniens) ont avancé, suivant Twardowski (1894).

12. Cf. Brentano (1982), p. 21 : «Les corrélats [de la paire intentionnelle acte-contenu] ne sont pas séparables l'un de l'autre, sauf de manière distinctionnelle ».

13. C'est le cas de Stumpf et de Marty. 
quement des actes ${ }^{14}$. C'est d'ailleurs Stumpf qui emploie l'expression «état de choses" de manière technique pour la première fois en 1888 afin de caractériser le contenu spécifique à l'acte de jugement ${ }^{15}$. Si l'expression est de sa plume, Stumpf souligne bien qu'elle n'exprime rien d'autre dans son usage que ce que Brentano appelait longtemps avant lui un contenu de jugement (Urteilsinhalt) immanent à celui-ci. Lorsqu'on voit ce concept d'état de choses comme fondateur de ses différentes variétés autrichiennes développées par la suite, le diagnostic proposé par Morscher exige des précisions supplémentaires:

As far as I can see, in most or all cases the dominant aim in introducing propositions and/or states of affairs [in 19th century Austrian philosophy] is to guarantee the independence from time, space, change, mind and language, of truth and falsity and of logical properties and relations like (in)validity, logical consequence, (in)compatibility and so on. In order to guarantee the objectivity of those attributes, the Austrian philosophers [...] thought that we need special truth-bearers and/or special truth-makers, because the "normal" truthbearers (like sentences and thoughts) and the "normal" truth-makers (the objects the sentences and thoughts are about) are or at least could be temporal and spatial, changeable and (in the case of truth-bearers like thoughts and sentences) also mind- or language-dependent [...] some Austrian philosophers wanted to guarantee the objectivity of truth-values and logical attributes via the objectivity of the truth-bearers, others via the objectivity of the truthmakers. Because of the convergence of aim this difference in the means was ignored by many Austrian philosophers, which led to confusions and misunderstanding among them. [...] [T] he views of Austrian philosophers on propositions and states of affairs were similar at a superficial level but more fundamentally in opposition ${ }^{16}$.

Si on suit ce diagnostic, il faudra expliquer en quoi l'introduction par Brentano du concept d'un contenu spécifique au jugement sert à expliquer l'objectivité des propriétés logiques et de la vérité et de la fausseté. Cette explication me semble ardue, car les contenus de jugement ne sont pour Brentano ni des vérifacteurs ni des porteurs de vérité objectifs au sens des propositions en soi de Bolzano.

Or, comme je veux le suggérer ici, il y a des raisons de douter que l'introduction de ce concept servait effectivement ce but au départ. Bien plus, la nécessité d'assurer l'objectivité des porteurs de vérité et/ou des vérifacteurs intervient plus tardivement dans la philosophie autrichienne. Ainsi, le diagnostic de Morscher quant à la confusion des Autrichiens sur les états de choses ne peut être redevable à une confusion des objectifs - celui d'assurer l'objectivité des porteurs de vérité par rapport à celui d'assurer l'objec-

14. C'est le cas de Husserl.

15. Cf. Stumpf (2006a), p. 287.

16. Morscher (1986), p. 80sq. 
tivité des vérifacteurs. La confusion sur la nature et le rôle des états de choses découle d'abord de la confusion quant à leur objectivité même, comme le montre cette portion de l'histoire des états de choses allant de Brentano à Stumpf.

\section{Contenu et objet des représentations}

C'est Twardowski qui introduit cette distinction en 1894, en s'inspirant d'abord d'une remarque de Höfler et de Meinong (1890). Curieusement, Meinong et Höfler réagissent très différemment à l'écrit de Twardowski. Höfler (1905) se félicite d'être effectivement reconnu comme l'instigateur de ce concept, alors que Meinong se montre plutôt reconnaissant envers Twardowski, car il admet ne jamais avoir fait cette distinction avant la publication de l'écrit de Twardowski ${ }^{17}$. C'est d'ailleurs cet ouvrage qui, l'année même de sa parution, pousse Husserl à revoir en détail plusieurs aspects de sa propre conception des contenus de représentations, révision qui le mènera à la conception présentée dans les Recherches logiques ${ }^{18}$.

Si Twardowski, Meinong, Husserl et Höfler acceptent cette distinction, elle ne fait pas pour autant l'unanimité au sein des membres de l'école de Brentano. Kastil (1909) s'en prend longuement à Twardowski et à Höfler, tandis que Kraus (1930: p. 192sq) renchérit contre Höfler, dans le même sens que Mayer-Hillebrand quelques années plus $\operatorname{tard}^{19}$. Pour Kraus, Kastil et Mayer-Hillebrand, il n'y a tout simplement aucune bonne raison de distinguer entre le contenu et l'objet des représentations si Brentano n'a jamais fait cette distinction.

Or pourquoi Brentano ne distinguerait-il pas, pour ce qui est de la représentation, entre le contenu de mon acte de représenter la tour Eiffel et la tour Eiffel elle-même? Il faut d'abord garder à l'esprit que la psychologie empirique de Brentano jouit d'une indépendance face à la métaphysique, et que c'est cette indépendance qui garantit à la première son statut de science. Elle est libérée de toute présupposition métaphysique quant à la nature et l'existence de l'âme et des objets du monde ${ }^{20}$. Conséquemment, les objets

17. Cf. Meinong (1899), p. 185 (trad.fr. (2007), p. 263) et Höfler (1905). Vu la caractérisation de l'objet de la représentation comme chose en soi kantienne (Höfler et Meinong (1890), p. 6), il est plausible de donner raison à Meinong contre la version höflerienne encore très répandue aujourd'hui, par exemple dans Jacquette (1990), p. 178; (2004), p. 105sq.; Smith (1994), p. 186; Benoist (2001a), p. 79; Cavallin (1997), p. 53; T n sescu (1998), p. 53; Chrudzimski (2001a), p. 120; (2002), p. 189; Hickerson (2005), p. 464.

18. Cf. Husserl (1990), daté de 1894.

19. Les travaux d'édition de Mayer-Hillebrand effectués notamment sur Brentano (1956) et (1959) attestent largement de son parti pris pour la position de Kraus et de Kastil.

20. Cf. Brentano (2008), p. 34. À partir de 1870, Brentano défend en fait une conception de la psychologie comme fondatrice pour la métaphysique où la première est détachable unilatéralement de la seconde, comme plusieurs passages de l'introduction de Brentano (2008) l'attestent. On retrouve aussi cette idée dans d'autres textes, par exemple Brentano (1982), 
externes, comme la tour Eiffel, n'occupent aucune place dans le schéma de la relation entre l'acte et le contenu (ou objet) de représentation.

On peut également s'assurer de cette absence de distinction entre contenu et objet, ou plus précisément de l'emploi synonymique des termes «contenu » et «objet» en partant des textes eux-mêmes. Le premier exemple, qui est peut-être aussi le plus éloquent, est à trouver dans le passage célèbre de la Psychologie d'un point de vue empirique où Brentano introduit l'intentionnalité comme caractéristique principale démarquant les phénomènes psychiques (représentations, jugements, émotions) des phénomènes physiques (les contenus de sensation en général) ${ }^{21}$. Brentano y emploie effectivement les expressions "relation à un contenu» (Beziehung auf einen Inhalt) et "direction vers un objet» (Richtung auf ein Objekt) de manière équivalente pour caractériser l'intentionnalité. Il est donc naturel de supposer que «Inhalt» et «Objekt» ont ici le même sens. À plusieurs endroits de la Psychologie, Brentano emploie le terme "Gegenstand " également comme synonyme de «Inhalt» ou "Objekt », là où les cas le permettent ${ }^{22}$. Bref, si on s'en tient à la Psychologie, il est difficile de soutenir que Brentano aurait effectivement en vue une distinction entre le contenu et l'objet des représentations.

On retrouve également des confirmations de l'emploi synonymique de ces deux ou trois termes dans les écrits de ses étudiants se basant sur les leçons de logique professées par le maître dans les années 1880. Le meilleur exemple est à trouver chez Hillebrand:

[c]e qui demeure spécifiquement le même si je me représente un thaler, un cheval ou un triangle, c'est l'acte de représentation; ce qui distingue ces trois cas les uns des autres est généralement désigné par contenu, objet [Gegenstand] ou objet de la représentation ${ }^{23}$.

Évidemment, cette citation ne fait qu'illustrer le caractère non problématique de l'emploi synonymique de "contenu» et «objet », elle ne répond pas à l'objection naturelle selon laquelle il semble bien y avoir une différence entre le contenu de ma représentation de la tour Eiffel et la tour Eiffel, cet objet parisien doté d'une quantité de propriétés matérielles totalement étrangères aux propriétés de mon contenu de représentation. Rappelons que Twardowski avait discuté ce point dans sa thèse d'habilitation en l'illustrant

p. 157. Cf. également la remarque introductive de la leçon de 1874-1875, Ausgewählte metaphysische Fragen, citée dans Werle (1989), p. 113.

21. Cf. Brentano (2008), p. 106.

22. Ibid., p. 107, 115, 136, 141 et passim.

23. «Dasjenige, was der Species nach gleichbleibt, ob ich einen Thaler, ein Pferd oder ein Dreieck vorstelle, heißt der Vorstellungsact; dasjenige, was diese drei Fälle von einander unterscheidet, wird allgemein als Inhalt, Gegenstand oder Object der Vorstellung bezeichnet". Cf. Hillebrand (1891), p. 37. À titre indicatif, voir aussi Stumpf (1890), p. 280, et Husserl (1891), p. 20, 45, 47, 157, 175 et passim. 
à l'aide de l'exemple du tableau ${ }^{24}$. Le tableau peint, par exemple le tableau de Brueghel intitulé Paysage d'hiver, est au contenu de la représentation ce que le paysage dépeint est à l'objet de la représentation. Avec cette analogie, Twardowski voulait mettre en valeur le fait qu'un seul et même acte de représentation représente en un double sens: l'acte de représenter a un contenu qui lui est immanent et qui est représenté en un sens déterminant de l'adjectif, et c'est sur cette base qu'un objet est représenté par le contenu, cette fois-ci dans un sens modifiant du même adjectif. En poursuivant l'analogie avec la peinture, on pourrait dire que l'acte de peindre se dirige d'abord vers le tableau comme objet matériel, la trame où sont agencées les couleurs, mais que ce tableau est un moyen de représenter quelque chose qui est à l'extérieur du tableau, à savoir le paysage en tant que tel.

L'exemple du tableau n'est toutefois qu'une illustration. Au départ, Twardowski introduit cette distinction entre le contenu et l'objet des représentations sur la base de la distinction entre contenu et objet du jugement acceptée par Brentano. Fidèle à son maître, Twardowski souligne que l'objet du jugement est ce qui est reconnu (anerkannt) ou rejeté (verworfen) - si je juge que A existe, c'est A qui est reconnu - mais que le contenu du jugement n'est pas A à proprement parler, mais bien l'existence (ou la non-existence) de A. En supposant une analogie parfaite entre la fonction du représenter et celle du juger et en soulignant que le contenu du jugement agit comme signe de l'objet, Twardowski en déduit qu'une même distinction s'impose dans la sphère du représenter ${ }^{25}$.

Le deuxième argument de Twardowski en faveur d'une distinction entre contenu et objet du représenter est basé sur une analogie exacte (genaues sprachliches Bild) entre noms et représentations ${ }^{26}$ : si on reconnaît aux noms les trois fonctions des représentations que sont la communication (Kundgebung), la signification (Bedeutung) et la nomination (Nennung), et qu'on reconnaît une analogie entre la pensée et la parole, on doit aussi reconnaître une analogie des fonctions pour ce qui est de la représentation: une expression " $\mathrm{A}$ » communique un acte de représentation (le représenté d'A), elle signifie un contenu (la représentation d'A), et elle nomme un objet (A).

L'argumentation de Twardowski par analogie n'est pas convaincante: l'analogie structurelle entre les fonctions représentatives et judicatives est simplement supposée, et rien n'est offert pour en montrer la validité. Twardowski appuie son analogie sur Marty (1884a: p. 293), mais précisément à la page citée par Twardowski, Marty souligne que deux noms peuvent avoir la même représentation. Si on part de Marty et de Brentano, il ne peut y avoir d'analogie exacte entre la structure et les fonctions du nom, et la struc-

24. Cf. Twardowski (1894), p. 13sq.

25. Ibid., p. 9.

26. Ibid., p. 10. 
ture et les fonctions des représentations. Même que toute la théorie des jugements existentiels de Brentano et l'idée de forme interne du langage développée par Marty visent à montrer que les noms et les énoncés ont des structures différentes de celles des représentations et des jugements. D'autre part, l'analogie entre les fonctions des expressions et celles des représentations présuppose que chaque nom remplit toutes ses fonctions, ce qui est difficile à dire pour des noms comme Sherlock Holmes ${ }^{27}$. Du coup, du point de vue des justifications psychologiques, le principe selon lequel chaque représentation a un contenu et un objet est installé sur des bases plutôt fragiles.

Le parallèle entre noms et représentations n'est donc pas en mesure d'appuyer la distinction contenu/objet des représentations de Twardowski. Les arguments sur lesquels il doit s'appuyer sont ceux développés dans la deuxième partie de l'ouvrage, et ils se basent tous sur la distinction de modes d'existence (l'être existant et l'être représenté) comme étant deux modes d'existence de plein droit pour les objets, à la manière de Meinong quelques années plus $\operatorname{tard}^{28}$. Si les motifs de cette distinction de Twardowski, quant aux représentations, prennent pour point de départ la conception brentanienne des représentations, leur justification, elle, est à trouver du côté d'une distinction ontologique entre différents modes d'existence.

\section{Contenu et objet des jugements}

Pourquoi Brentano aurait-il besoin d'une distinction entre les contenus et les objets des jugements s'il peut se passer de cette distinction en ce qui concerne les représentations? Les raisons sont nombreuses. La première, qui est aussi la plus importante, est aussi la plus simple; Brentano distribue l'ensemble des phénomènes psychiques en trois classes: les représentations constituent la classe fondatrice, à laquelle s'ajoute celle des jugements et des actes affectifs et volitifs. Ces deux dernières classes sont fondées sur la première, mais cela ne signifie pas qu'un jugement est décomposable en représentations. Lorsque Brentano affirme que l'acte de jugement présuppose un acte de représentation, il veut simplement dire que, pour juger que A est, je dois avoir représenté A préalablement. La relation entre l'acte de représentation et l'acte de jugement en est une de détachabilité unilatérale (dans l'unité de la conscience, l'acte de représentation est détachable de l'acte de jugement, mais non l'inverse $)^{29}$. Ce qui n'empêche pas que l'acte de jugement en tant que tel soit un phénomène complet en soi. Il n'est pas décomposable en une représentation à laquelle on aurait ajouté quelque chose de plus ${ }^{30}$. Or ce qui caractérise le jugement comme phénomène psychique est la reconnaissance

27. Je remercie Wolfgang Künne d'avoir attiré mon attention sur ce point.

28. Ibid., p. 29 sq.

29. Cf. Brentano (1982), p. 12.

30. Brentano (2008), p. 227sq. offre plusieurs arguments contre les conceptions du jugement en terme de composition de contenus de représentation. 


\section{0 • Philosophiques / Printemps 2011}

ou le rejet de l'existence d'un objet: l'objet du jugement A est donc A, mais le contenu du jugement ne peut être A également; car le jugement n'est pas seulement reconnaissance et rejet de A, mais il est reconnaissance et rejet de l'existence de A. L'existence n'étant pas une propriété d'objet selon Brentano, elle intervient sur le plan du contenu.

Une autre motivation de Brentano à distinguer entre le contenu et l'objet du jugement repose sur sa conception corrélative des actes de conscience. Les phénomènes psychiques ont des structures relationnelles; chaque acte se rapporte à un contenu, et les parties du phénomène (acte et contenu) sont corrélatives: une ne peut exister sans l'autre, et vice-versa. La paire de corrélats acte/contenu a ceci de particulier que seul le premier corrélat est réel (l'acte) alors que le second est non réel. Une autre particularité de cette paire de corrélats est que ceux-ci sont des parties distinctionnelles d'un tout qui est le phénomène psychique. Or, si on peut distinguer seulement conceptuellement le contenu d'un acte de représentation, en quoi ce même contenu pourrait être exactement le même que celui d'un acte de jugement? L'acte de représentation et l'acte de jugement étant deux actes différents, les parties de l'un ne peuvent entrer dans les parties de l'autre.

Le concept d'existence, comme tous les autres concepts, est obtenu par réflexion sur nos actes concrets de jugement. C'est l'analyse des actes de jugements qui nous permet d'isoler ce concept. Cette thèse, on la retrouve dans les ouvrages de Brentano ainsi que dans plusieurs œuvres de ses élèves ${ }^{31}$. Dans la Psychologie d'un point de vue empirique, Brentano expose cette thèse de la manière suivante:

Certains ont pensé que ce concept [=le concept d'existence] ne pouvait être tiré de l'expérience. Nous aurons donc à l'examiner à ce point de vue quand nous traiterons des idées dites innées. Et nous trouverons alors qu'il provient bien de l'expérience, mais de l'expérience interne, et qu'il n'a été obtenu qu'en vue du jugement. Pas plus que le concept de jugement, le concept d'existence n'a donc pu être prédicat dans le premier jugement. Et l'on reconnaît également de cette façon que du moins la première perception, celle qui était donnée dans le premier phénomène psychique, ne peut avoir consisté en un tel jugement prédicatif $^{32}$.

Bref, pour en arriver à distinguer les différentes parties d'un acte de jugement, nous devons recourir à la perception interne (ce que Brentano appelle ici l'expérience interne), et c'est en partant de la perception interne - la perception que nous avons de nos actes de jugement, que nous pouvons obtenir le concept d'existence. Autrement dit, l'analyse proposée va du

31. Par exemple Brentano (1930), p. 45; Marty (1884), p. 171sq.; Hillebrand (1890), p. 27.

32. Brentano (1944), p. 215; (2008), p. 233 (traduction française modifiée). 
jugement vers le concept d'existence, et non l'inverse, comme c'est le cas pour la plupart des théories propositionnelles du jugement ${ }^{33}$.

Le concept d'existence et le plan sur lequel il intervient dans l'analyse selon Brentano est intéressant pour la raison que la question même de l'existence ou de la non-existence de certains objets de représentation, les centaures et les cercles carrés par exemple, devient un non-lieu lorsqu'on aborde le problème strictement sur le plan de la représentation. Car si on en reste sur ce plan, rien dans la représentation ne nous donne accès, pour ainsi dire, au concept d'existence. Autrement dit, un agent dénué de capacité judicative ne serait pas en mesure de statuer sur la question des représentations sans objet $^{34}$. Cela explique pourquoi la catégorie d'objet devient équivoque seulement à partir du jugement: lorsque je me représente la table devant moi, puis que je juge qu'elle existe, l'objet demeure le même, mais lorsque je me représente un centaure, puis que je juge qu'il n'existe pas, l'objet de mon jugement ne peut être celui de ma représentation initiale, puisque je rejette précisément qu'il y ait une telle chose. Puisque les jugements sont des reconnaissances ou des rejets, il doit bien y avoir quelque chose de reconnu ou de rejeté. Au cours de sa carrière, Brentano a proposé plusieurs différentes explications de ce problème, mais à mon avis aucune n'est vraiment satisfaisante: certaines d'entre elles consistent à expliquer le rejet du centaure par le rejet d'une ens rationis (le centaure-représenté), d'autres par le rejet d'états de choses négatifs (la non-existence du centaure) ${ }^{35}$. En 1911, le recours aux entités négatives est justifié de la manière suivante: un jugement existentiel négatif comme «il n'y a pas de centaures» reviendrait à affirmer que quelqu'un qui juge qu'il y a des non-centaures juge correctement. Les réalités négatives qui sont ainsi supposées sont essentiellement des fictions, et il met en relation ce recours à des entités négatives à l'emploi de l'onoma aoriston chez Aristote ${ }^{36}$. Bref, le seul changement notable dans la conception brentanienne des jugements existentiels négatifs vrais entre la période de jeunesse et la maturité est que Brentano semble parfois reconnaître des entités intentionnelles, mais que plus tard il réduit leur existence à un simple nom ou un suppositum, quelque chose qui pose pour objet mais qui n'en est pas un. Cette solution proposée en 1911 n'est pas foncièrement différente de

33. La même idée est défendue aussi plus tard, dans les cours de logique de Vienne. Cf. Brentano (1956).

34. Sur la formulation de ce problème chez Bolzano et sa réception chez Twardowski et Husserl, cf. Fréchette (2010).

35. Sur la dernière explication, $c f$. Brentano (1930), p. 133. Cf. également Srzednicki (1965), p. 79.

36. Cf. Brentano (1944), p. 290; (2008), p. 417. Même si sa conception de la négation lui aurait permis de s'en passer, Bolzano a lui aussi fait une place dans sa théorie des représentations à la représentation purement négative (rein verneinende Vorstellung) exprimée par un onoma aoriston, sous-catégorie des représentations sans objet. Cf. Bolzano (1837), p. 416sq, 421 (tome I); p. 48 (tome II); p. 220sq. (tome IV). 
celle qu'il esquisse en 1885 dans le Logikkolleg, où il avance la thèse que ces jugements n'ont tout simplement pas d'objet ${ }^{37}$. D'une manière ou d'une autre, Brentano n'a jamais été tout à fait satisfait des conséquences de sa théorie du jugement en ce qui concerne les jugements existentiels négatifs vrais et la question de leur objet. Mais il n'en reste pas moins qu'il a toujours fait de ce cas un cas d'exception dans sa théorie du jugement, et non un cas paradigmatique $^{38}$.

Pour simplifier ce que je viens d'exposer ici, je propose de schématiser la distinction entre le contenu et l'objet des jugements de la manière suivante:

$\begin{array}{lll}\text { actes } & \text { contenus } & \text { objets } \\ \text { représentations } & \mathrm{cR} & \mathrm{oR} \\ \text { jugements } & \mathrm{cJ} & \mathrm{oJ}\end{array}$

Théoriquement, les distinctions entre contenus et objets peuvent se faire à la fois de manière verticale et de manière horizontale. Husserl, par exemple, remplit les quatre cases avec quatre entités distinctes: le contenu de la représentation (cR) est une signification différente de son objet (oR), le contenu du jugement est une proposition (cJ) différente de l'état de chose qui la rend vraie (oJ). Chez Twardowski (1894), les distinctions horizontales sont faites, mais on ne trouve seulement qu'une des deux distinctions verticales, celle des contenus ${ }^{39}$.

Chez Brentano, la distinction horizontale est effectivement faite en ce qui a trait au jugement, mais elle n'est pas faite en ce qui concerne la représentation. Autrement dit, $c R$ et oR ne sont pas distingués. Si j'ai raison de dire que $\mathrm{cR}$ et oR ne sont pas distingués, que ces deux appellations désignent la même chose, alors il suffit simplement de montrer que cJ est distinct de $\mathrm{cR}$ pour montrer que non seulement cJ est distinct d'oJ, mais aussi qu'oR est distinct d'oJ. C'est pourquoi j'ai d'abord tenté de montrer que le contenu et l'objet de la représentation sont pour Brentano une seule et même chose, et que j'ai ensuite montré pourquoi le contenu du jugement et le contenu de la représentation ne sont pas la même chose; il en résulte que l'objet de la

37. Cf. Brentano EL81, p. 13515: «On doit distinguer entre 1) l'objet de la représentation et la représentation [...], 2) tout comme entre l'objet du jugement et le jugement [...]. Les premiers font souvent défaut (comme dans le jugement existentiel négatif vrai» ([m]an muss unterscheiden 1) zw[ischen] dem Object d[er] Vorstell[un]g u[nd] der Vorstell[un]g [...] 2) ebenso zw[ischen] d[em] Object d[es] Urtheils u[nd] dem Urtheil [...] Die ersteren fehlen oft. ([wie] beim wahren negativen Urtheil). Cf. aussi Brentano, EL81, p.13550.

38. Pour d'autres interprétations de la théorie des contenus de jugement $c f$. par exemple Mayer-Hillebrand (1959), p. 320sq.

39. La distinction verticale en ce qui concerne les objets est faite dans Twardowski (1996), p. 164; (2007), p. 351. Selon Betti (2005), Twardowski l'aurait faite quelques mois après la parution de Twardowski (1894). 
représentation ne peut être la même chose que l'objet du jugement. Naturellement, cela n'exclut pas la possibilité théorique que Brentano puisse accepter des objets complexes, des états de choses, tombant sous oJ. Les objets de jugements existentiels négatifs vrais seraient justement ce genre de candidats. Mais dans ce cas, pour maintenir la distinction entre cJ et oJ, ces états de choses devraient être d'une autre nature que cJ. Or Brentano ne semble pas avoir exploré cette voie dans ses écrits.

L'interprétation de la distinction entre oR et $\mathrm{oJ}$ que je suggère ici va plutôt à l'inverse de cette possibilité théorique. Selon cette interprétation, oR est distinct d'oJ dans tous les cas sauf dans le cas de jugements existentiels négatifs vrais ${ }^{40}$. Cela reviendrait à dire que dans le cas des jugements existentiels négatifs vrais, $c R$ prend la place d'oJ. Mais dans tous les autres cas, oJ est différent d'oR. Si je me représente la table devant moi, puis je juge que la table existe, la différence entre oR et oJ est tout simplement celle entre le contenu de la représentation et l'objet qui est jugé. Cette interprétation me semble rendre compte de la manière dont Brentano conçoit l'objet des jugements en mettant en relief le cas d'exception que constituent chez Brentano les jugements existentiels négatifs vrais.

\section{La distinction entre objectif et réel}

Jusqu'à présent, j'ai insisté d'une part sur le fait que Brentano distingue effectivement entre le contenu de jugement et le contenu de représentation (les contenus sont constitués différemment, le premier est généralement de la forme «l'existence de $\mathrm{A}$ », le second de la forme « $\mathrm{A}$ »), mais d'autre part sur le fait que la spécification d'un contenu de jugement à la fois distinct du contenu de représentation et de l'objet du jugement découle d'une motivation psychologique, et que sa justification aussi est psychologique et non ontologique. Autrement dit, les contenus spécifiques au jugement sont justifiés par le caractère irréductible du jugement à la représentation. Ils ne suivent pas la motivation que Morscher voit comme caractéristique de presque toute la philosophie autrichienne, celle d'assurer l'objectivité des propriétés logiques et de la vérité et de la fausseté. En fait, comme nous l'avons vu, les contenus de jugement de Brentano ne sont pas de forme propositionnelle et ils ne sont pas ontologiquement indépendants des actes dont ils sont les contenus.

On peut naturellement objecter que même si leur forme non propositionnelle et leur dépendance ontologique face aux actes empêchent les contenus de jugement (et de représentation) d'être des candidats aux tâches identifiées par Morscher, il faut cependant expliquer ce qui distingue onto-

40. J'insiste ici sur les jugements existentiels négatifs vrais, car quelqu'un qui juge qu'il n'y a pas de lions émet selon Brentano un jugement dénué d'évidence, un jugement à proprement parler aveugle, ce qui implique donc que l'objet représenté dans le jugement n'est pas celui qui est rejeté. 
logiquement les contenus des actes, puisque l'analyse descriptive menée par Brentano les distingue effectivement.

$\mathrm{Au}$ cours de sa carrière, Brentano a proposé plusieurs explications de cette distinction. Dès 1862, il se montre préoccupé par la défense d'une conception homonymique (et non plurivoque) du terme «être» chez Aristote ${ }^{41}$. Ce qui est alors déterminant pour lui d'un point de vue métaphysique, c'est l'être selon les catégories. Et de ce point de vue - c'est l'essentiel de sa thèse -, les catégories aristotéliciennes sont complètes. Du point de vue de la théorie de la connaissance et de la psychologie, c'est l'être au sens du vrai (das Seiende als Wabre), compris comme une détermination mentale de l'être, qui est déterminant, et ce dernier n'est pas du ressort de la métaphysique $^{42}$. De cette distinction dépend celle entre concepts réels, relevant des catégories, et concepts objectifs: l'être au sens du vrai est compris comme un concept objectif, l'expression ne signifiant rien d'autre que quelque chose qui n'a de réalité que dans l'entendement ou l'esprit ${ }^{43}$. Le «est » du jugement relève en ce sens de l'être au sens du vrai:

[T]oute chose de la pensée, c'est-à-dire tout ce qui, existant objectivement dans notre esprit, peut devenir le sujet d'un énoncé affirmatif vrai, appartiendra [à l'être au sens du vrai]. Rien de ce que nous formons dans notre esprit n'est à ce point dénué de réalité qu'il faille l'exclure totalement du domaine de l'être au sens du vrai $[\ldots]^{44}$.

Ce que Brentano reprend ici d'Aristote, la distinction entre le réel et l'objectif, est une première version de la distinction qu'on retrouvera dans la Psychologie de 1874 entre le réel et l'intentionnel, puis de celle présente dans les derniers écrits de Brentano entre le réel et l'irréel. Il s'agit naturellement d'une distinction ontologique, mais on doit toutefois souligner que la séparation entre être mental et être réel suit celle entre, respectivement, la théorie de la connaissance et la psychologie d'une part, puis la métaphysique d'autre part. Dès ses premiers écrits et jusqu'à la fin de sa vie, Brentano a voulu tenir distincts les domaines de recherche de la métaphysique et de la théorie de la connaissance, dont la tâche incombe à la psychologie. En ce sens, l'être objectif identifié dès la thèse de 1862 ne doit pas être considéré comme une sorte d'existence diminuée vis-à-vis de l'être selon les catégories, mais plutôt comme une façon de caractériser l'objet d'étude de la psychologie: les actes mentaux et leur contenu.

41. Cf. Brentano (1862).

42. Ibid., p. 14.

43. Ibid., p. 82.

44. Cf. Brentano (1862), p. 37. Traduction française modifiée de Brentano (1992), p. 49. Brentano ne nie pas qu'Aristote admet différents sens de la vérité ou de la fausseté, mais en préconisant les exposés du quatrième livre de la Métaphysique d'Aristote (1012b8), il donne préséance à la thèse selon laquelle le vrai n'est rien d'autre qu'une affirmation et le faux une négation. Cf. Brentano (1862), p. 21; (1992), p. 37. 
Cinq ans plus tard, dans sa thèse d'habilitation, cette opposition entre le réel et l'objectif n'a pas fondamentalement changé, et elle est encore à comprendre dans le même sens que celui exposé en 1862:

Nous employons ici l'expression "objectif» [...] dans le sens que les aristotéliciens du Moyen Âge lui ont adjoint (l'«objectif» scolastique) et qui permet une caractérisation très courte et précise de la doctrine d'Aristote. Matériellement, comme propriété physique, le froid est dans ce qui est froid; comme objet, c'est-à-dire en tant que ce qui est senti, le froid est dans celui qui ressent le froid ${ }^{45}$.

Autrement dit, la froideur est matériellement dans ce qui est froid: elle est dans le bloc de glace; mais objectivement elle est dans celui qui ressent le froid. La distinction matériel/objectif (que Brentano thématise aussi parfois par réel/objectif, réel/intentionnel, ou plus tard par réel/irréel) s'applique naturellement aussi sur le plan des relations: la ressemblance structurelle entre les prédicats «...est matériellement dans_» et "...est objectivement dans_" est trompeuse, car la relation d'inclusion dans le premier cas est une relation réelle, dans le second une relation «modifiée» ou «objective».

On retrouve aussi cette conception dans les leçons professées par Brentano à cette époque. La question de la substance et de sa nature relève pour Brentano de la métaphysique et de l'être au sens matériel et propre (sachlich Seiendes), ce n'est pas là une question qui intéresse le logicien ou le psychologue. Plusieurs passages des leçons de métaphysique de Würzburg datées de 1867 attestent de cette distinction. Citons seulement les deux suivants :

Avant tout, il est clair que nous devons distinguer deux êtres (Seiendes), desquels Aristote a déjà spécifié les significations en distinguant entre l'on es alethes et l'on eo tes dianoias. L'être est d'abord employé au sens du vrai [comme caractérisation de l'assentiment], [expression de l'affirmation], [c'est-à-dire comme moyen de caractériser que quelque chose est vrai], puis ensuite au sens de l'être concret (sachlich), donc dans sa signification logique et réelle ${ }^{46}$.

\section{L'être au sens du vrai, poursuit Brentano}

n'est pas en fait l'objet de la métaphysique, car celle-ci n'a pas à traiter de ce qui se trouve spécifiquement dans notre esprit. Elle doit plutôt traiter de toutes les choses en général et en connaître les causes [le logicien et en partie le psychologue en parle ex professo $]^{47}$.

45. Brentano (1867), p. 82.

46. Brentano M96, p. 31948. Cité selon Antonelli (2001), p. 255 : «Vor allem ist es klar, dass wir ein zweifaches Seiendes unterscheiden muessen, dessen Bedeutungen schon Aristoteles gesondert hat, indem er das on es aletes von dem on eo tes dianoias schied. Das Seiende wird gebraucht einmal <als Bezeichnung der Zustimmung $>$, < Ausdruck der Bejahung > im Sinne des Wahren (d.h. als Bezeichnung dafür, dass etwas wahr sei>, dann im Sinne des Sachlichen Seienden, also in einer logischen und realen Bedeutung". Pour d'autres passages, cf. Ibid., p. 254.

47. Ibid. (cité selon Antonelli (2001), p. 259: «ist nicht eigentlich Gegenstand der Metaphysik, denn diese hat ja nicht von etwas was speciell in unserm Geiste ist, sondern im Allge- 
La période de maturité ne fait pas exception à cette opposition entre l'objectif et le réel, comme on peut le voir dans les textes en annexe de Brentano (1911). On peut voir dans ces textes non seulement que Brentano maintient toujours la distinction entre contenus de jugement et contenus de représentation, mais surtout que l'être des contenus de pensée est toujours conçu comme un être impropre:

[C]'est notamment à propos de l'activité psychique qui se manifeste dans le jugement qu'on distingue l'objet et le contenu du jugement. Dans un jugement tel que «Il n'y a pas de centaures ", le centaure serait l'objet, mais le contenu du jugement serait l'inexistence de ce centaure. Quand on dit que ce contenu est dans l'agent psychique, on fait un nouvel emploi impropre du terme «être"; si l'on veut garder à ce terme son véritable sens, il faut dire, ce qui revient au même, qu'un agent psychique est actuellement en train de nier un centaure ${ }^{48}$.

Voici donc ce que nous considérons comme établi: l'être ou le non-être d'un centaure ne peuvent devenir objets au sens où pourrait le devenir le centaure lui-même; seuls peuvent devenir objets le sujet qui affirme et celui qui nie le centaure, auquel cas le centaure devient également objet selon un certain modus obliquus ${ }^{49}$.

En ce sens, la distinction entre l'être au sens du vrai (l'être objectif) et l'être réel est maintenue même après le tournant réiste. Ce qui change après ce tournant, c'est que des façons de parler comme «Anne a présentement pour contenu de jugement l'inexistence d'un centaure» sont systématiquement traduites par "Anne est présentement en train de nier un centaure ". Mais l'être des contenus de jugement est toujours considéré comme un être au sens impropre. À mon avis, cette distinction entre ce qui est objectif (ou impropre, intentionnel, vrai, etc.) et ce qui est réel (ou propre, concret, etc.) délimite ce qui est du ressort de la psychologie et ce qui est du ressort de la métaphysique. Si on garde à l'esprit que la métaphysique est fondée par la psychologie selon Brentano, on peut alors comprendre comment Brentano peut distinguer ontologiquement entre l'acte et le contenu: dans les termes de l'ontologie monocatégorielle réiste par exemple, l'acte appartient toujours à la sous-classe des entités étendues uniquement temporellement, alors que ce qui est appelé «improprement» le contenu peut appartenir soit à la même sous-classe, soit à la sous-classe des entités étendues spatiotemporellement (les corps). Dans les termes de l'ontologie des irrealia, l'acte est l'accident d'une substance (le sujet pensant), et le contenu est une partie non réelle de cet accident. Si cette distinction est une distinction ontologique, elle est toutefois possible uniquement sur la base de la psychologie.

meinen von allen Dingen zu handeln und sie aus ihren Gruenden zu erkennen. $<$ Der Logiker und zum Theil der Psychologe sprechen ex professo von ihm>».

48. Brentano (2008), p. 159; (1944), p. 284.

49. Brentano (2008), p. 162; (1944), p. 286. 


\section{Remarques finales}

Ce que j'ai voulu proposer ici, c'est en quelque sorte un diagnostic de remplacement de celui de Morscher sur l'introduction des états de choses dans la philosophie autrichienne. En introduisant des contenus de jugement, Brentano ne visait pas à garantir l'objectivité des propriétés logiques ou l'indépendance ontologique de la vérité ou de la fausseté. L'introduction des contenus de jugement chez Brentano découle de sa théorie des jugements existentiels, une théorie basée d'une part sur une thèse empiriste quant à l'origine des concepts - même le concept d'existence découle de la réflexion sur des actes de jugement concrets - , et d'autre part sur une conception de la vérité en termes d'évidence.

Naturellement, cette motivation empiriste et psychologique de l'introduction des contenus de jugement n'exclut pas que, partant de cette motivation psychologique, on puisse apporter en plus des justifications ontologiques à la distinction entre l'acte, le contenu et l'objet des représentations et des jugements. C'est ce que j'ai voulu illustrer en discutant des motivations et des arguments qui soutiennent la distinction entre le contenu et l'objet des représentations chez Twardowski. Mais comme on l'a vu, Brentano ne peut s'accorder avec les arguments employés par Twardowski.

Néanmoins, la nature des contenus de jugement brentaniens reste problématique. Comme on l'a vu, la distinction entre contenu de représentation et contenu de jugement fonctionne dans le cas des jugements existentiels affirmatifs vrais: la représentation de A et le jugement énoncé par «A est » ont, selon la théorie brentanienne, des contenus différents, et seul le jugement, et non la représentation, a un objet. Mais dans le cas des jugements existentiels négatifs vrais, qui est le cas problématique, c'est le contenu de la représentation qui agit comme objet du jugement. Bref, pour maintenir les distinctions que nous avons présentées dans la section 4, Brentano est forcé de donner aux contenus un rôle ontologique dans le cas des jugements existentiels négatifs vrais. Toutefois, comme je l'ai suggéré, je crois qu'il est plus charitable de voir le cas des jugements existentiels négatifs vrais comme un cas d'exception de la théorie de Brentano plutôt que comme un cas paradigmatique. Les nombreux et différents efforts qu'il a livrés au cours de sa carrière afin de pallier ce problème me semblent plaider en faveur de cette interprétation. En effet, il a envisagé dans les années 1880 que ces jugements soient tout simplement dénués d'objet; à peu près au même moment, mais aussi plus tard, il a proposé la thèse que c'est le nom du contenu qui agit comme suppositum pour l'objet du jugement négatif vrai et non comme objet $^{50}$. Et dans le dernier paragraphe de la dernière citation, il propose de réduire toutes les expressions dénotant un contenu à des expressions déno-

50. Le nom du contenu étant alors pris au sens d'un onoma aoriston, une fiction du langage, cf. Brentano (2008), p. 417; (1944), p. 290. Hillebrand (1891), p. 98, se basant sur les cours de logique de Brentano des années 1880, va exactement dans ce même sens. 
tant des agents produisant des actes mentaux. Des stratégies de ce genre font généralement partie de l'arsenal des nominalistes. C'est pourquoi il me semble que l'introduction du concept d'état de choses dans la philosophie autrichienne témoigne des interrelations fécondes entre les positions réalistes et nominalistes ${ }^{51}$.

\section{Références bibliographiques}

Antonelli, Mauro. Seiendes, Bewußtsein und Intentionalität im Frühwerk Franz Brentanos, Freiburg, Alber, 2001.

Benoist, Jocelyn. Représentations sans objets. Aux origines de la phénoménologie et de la philosophie analytique, Paris, Presses Universitaires de France, 2001.

Bergmann, Hugo. Untersuchungen zum Problem der Evidenz der inneren Wabrnehmung, Halle, Niemeyer, 1908.

Betti, Arianna. "Propositions et états de choses chez Twardowski», in Dialogue, vol. 46, 2005, p. 469-492.

Bolzano, Bernard. Wissenschaftslehre, Sulzbach, Seidelsche Buchhandlung (en 4 tomes), 1837.

Brentano, Franz. Von der mannigfachen Bedeutung des Seienden nach Aristoteles, Freiburg, Herder'sche Verlagshandlung, 1862.

- Die Psychologie des Aristoteles, insbesondere seine Lehre vom nous poietikos, Franz Kirchheim, 1867.

- Von der Klassifikation der psychischen Phänomene, Leipzig, Duncker \& Humblot, 1911 (reproduit dans Brentano, 2008).

—. Wabrheit und Evidenz, Leipzig, Meiner, 1930.

—. Psychologie du point de vue empirique, Paris, Aubier, 1944. (Réédition revue par J.-F. Courtine, Paris, Vrin, 2008). Trad. fr. de Brentano, 2008.

Brentano, Franz. Die Lehre vom richtigen Urteil, Bern, Francke Verlag, 1956.

—. Grundzüge der Ästhetik, Bern, Francke Verlag, 1959.

. Deskriptive Psychologie, Hamburg, Meiner, 1982.

- Aristote. Les diverses acceptions de l'être, Paris, Vrin, 1992 ( $2^{\mathrm{e}}$ édition 2005 , trad. fr. de Brentano, 1862).

- Psychologie vom empirischen Standpunkt, (M. Antonelli dir.), Francfort, Ontos Verlag, 2008.

- Schriften zur Sinnespsychologie (W. Baumgartner, dir.), Francfort, Ontos Verlag, 2009.

- Manuscrits inédits catégorisés selon le catalogue de Mayer-Hillebrand, 1951.

EL 81 Fragmente; M 96 Ontologie (Metaphysik).

Cavallin, Jens. Content and Object. Husserl, Twardowski and Psychologism, Dordrecht, Kluwer, 1997.

Chrudzimski, Arkadiusz. Intentionalitätstheorie beim frühen Brentano, Dordrecht, Kluwer, 2001.

—. "Die Intentionalitätstheorie Anton Martys", in Grazer Philosophische Studien, vol. 62, 2001a, p. 175-214.

51. Je remercie Claude Panaccio et Kevin Mulligan pour leurs remarques sur une première version de ce texte, ainsi que Denis Fisette pour ses commentaires. Cet article a bénéficié du soutien financier du Conseil de recherche en sciences humaines du Canada (CRSH). 
—. «Die Theorie der Intentionalität Meinongs», in Dialectica, vol. $55 \mathrm{n}^{\circ} 2$, 2001b, p. 119-143.

- . "Von Brentano zu Ingarden. Die phänomenologische Bedeutungslehre» in Husserl Studies, vol. 18, no 3, 2002, p. 185-208.

Chrudzimski, Arkadiusz et Barry Smith. "Brentano's Ontology : from Conceptualism to Reism ", in D. Jacquette (dir.), The Cambridge Companion to Brentano, Cambridge, Cambridge University Press, 2004, p. 197-219.

Fisette, Denis et Guillaume Fréchette. "Le legs de Brentano", in Denis Fisette et Guillaume Fréchette (dir.), À l'école de Brentano. De Würzburg à Vienne, Paris, Vrin, 2007, p. 13-160.

Fréchette, Guillaume. Gegenstandslose Vorstellungen. Bolzano und seine Kritiker, Sankt-Augustin, Academia, 2010.

Hickerson, Ryan. "Getting the Quasi-Picture: Twardowskian Representationalism and Husserl's Argument Against It ", in Journal of the History of Philosophy, vol. 43, n 4, 2005, p. 461-480.

Hillebrand, Franz. Die neuen Theorien der kategorischen Schlüsse, Vienne, Hölderm, 1891.

Höfler, Alois et Alexius Meinong. Philosophische Propädeutik, Theil 1: Logik, Vienne, Tempsky, 1890.

Höfler, Alois. "Sind wir Psychologisten ?", in Atti del V Congresso Internazionale di Psicologia tenuto in Roma dal 26 al 30 Aprile 1905, Rome, Forzani, 1905, p. 188-193.

Husserl, Edmund. Philosophie der Arithmetik. Psychologische und logische Untersuchungen, erster Band, Halle, Pfeffer, 1891 (rééd. La Haye, Martinus Nijhoff, 1970).

- 1900. Logische Untersuchungen Band 1. Prolegomena zur reinen Logik. Text der ersten und zweiten Auflage, La Haye, Martinus Nijhoff [1975].

_. "Husserls Abhandlung 'Intentionale Gegenstände' » (K. Schuhmann, dir.), in Brentano Studien, vol. 3, 1990, p. 142-176.

Jacquette, Dale. «The Origins of Gegenstandstheorie: Immanent and Transcendent Intentional Objects in Brentano, Twardowski, and Meinong" in Brentano Studien, vol. 3, 1990, p. 177-202.

- «Brentano's Concept of Intentionality », in D. Jacquette (dir.), The Cambridge Companion to Brentano, Cambridge, Cambridge University Press, 2004, p. 98-130.

Kastil, Alfred. Studien zur neueren Erkenntnistheorie, Halle, Max Niemeyer, 1909.

- Die Philosophie Franz Brentanos. Eine Einführung in seine Lehre, Bern, Francke Verlag, 1951.

Kraus, Oskar. "Brentanos Scheidung von deskriptiver und genetischer Psychologie und sein Verhältnis zur Phänomenologie und Gegenstandstheorie », in Brentano, Franz, Psychologie vom empirischen Standpunkt. Erster Band (O. Kraus, dir.), Leipzig, Meiner, 1924, p. xvii-xxiii.

- . "Anmerkungen des Herausgebers ", in Brentano, Franz, Wabrheit und Evidenz, Leipzig, Meiner, 1930, p. 165-220.

- Werttheorien. Geschichte und Kritik, Vienne, Rohrer, 1937.

Marty, Anton. «Über subjektlose Sätze und das Verhältnis der Grammatik zur Logik und Psychologie. Zweiter Artikel », in Vierteljahrsschrift für wissenschaftliche Philosophie, vol. 8, 1884, p. 161-192. Reproduit dans Marty, Anton, Gesam- 
melte Schriften, vol. II/1, Schriften zur deskriptiven Psychologie und Sprachphilosophie (J. Eisenmeier, dir.), Halle, Niemeyer, 1918, p. 36-62.

"Über subjektlose Sätze und das Verhältnis der Grammatik zur Logik und Psychologie. Dritter Artikel ", in Vierteljahrsschrift für wissenschaftliche Philosophie, vol. 8, 1884a, p. 292-340. Reproduit dans Anton Marty, Gesammelte Schriften, vol. II/1, Schriften zur deskriptiven Psychologie und Sprachphilosophie (J. Eisenmeier, dir.), Halle, Niemeyer, 1918, p. 62-101.

- Untersuchungen zur Grundlegung der allgemeinen Grammatik und Sprachphilosophie. Band I, Halle, Max Niemeyer, 1908.

Mayer-Hillebrand, Franziska. Verzeichnis der Manuskripte Franz Brentanos, Typoscript, Harvard, Houghton Library, 1951.

—. «Franz Brentanos ursprüngliche und spätere Seinslehre und ihre Beziehungen zu Husserls Phänomenologie », in Zeitschrift für philosophische Forschung, vol. 13, no 2, 1959, p. 316-339.

Meinong, Alexius. «Über Gegenstände höherer Ordnung und deren Verhältnis zur inneren Wahrnehmung» in Zeitschrift für Psychologie und Physiologie der Sinnesorgane, vol. 21, 1899, p. 182-272.

—. Über Annahmen (2e édition augmentée), Leipzig, Barth, 1910.

—. "Sur les objets d'ordre supérieur et leur rapport à la perception interne ", in D. Fisette et G. Fréchette (dir.), À l'école de Brentano. De Würzburg à Vienne, Paris, Vrin, 2007, p. 261-342 (trad. fr. de Meinong [1899]).

Morscher, Edgar. "Propositions and States of Affairs in Austrian Philosophy before Wittgenstein », in Nyíri, J.-C. (dir.), Von Bolzano zu Wittgenstein. Zur Tradition der österreichischen Philosophie, Vienne, Hölder, 1986, p. 75-85.

Mulligan, Kevin, Peter Simons, et Barry Smith. "Truth-Makers », in Philosophy and Phenomenological Research, vol. 44, 1984, p. 287-321.

Mulligan, Kevin. "'Wie die Sachen sich zueinander verhalten'. Inside and Outside the Tractatus", Theoria, vol. 5, n 2, 1985, p. 145-174.

"Judgings: Their Parts and Counterparts ", in Topoi, vol. 6 n 1 (supplément $\mathrm{n}^{\circ} 2$ intitulé La scuola di Brentano), 1988, p. 117-144.

—. "Post-Continental Philosophy: Nosological Notes", in Stanford French Review (numéro spécial intitulé Philosophy and the Analytic-Continental Divide, P. Engel, dir.) vol. 17, n 2-3, 1993, p. 133-150.

—. "How Perception Fixes Reference ", in Axel Burri (dir.), Sprache und Denken. Language and Thought, Berlin, Walter de Gruyter, 1997, p. 122-138.

- - "Sur l'histoire de l'approche analytique de l'histoire de la philosophie: de Bolzano à Brentano à Bennett et Barnes ", in J.-M. Vienne (dir.), Philosophie analytique et histoire de la philosophie, Paris, Vrin, 1997, 1997a, p. 61-103.

_. "Exactitude et bavardage. Gloses pour une opposition paradigmatique dans la philosophie autrichienne", in Philosophiques, vol. 26, n 2, 1999, p. 177-201.

-. "C'était quoi la philosophie analytique?", in K. O. Apel, J. Barnes et al. (dir.), Un siècle de philosophie 1900-2000, Paris, Gallimard, 2000, p. 332-366.

__. "De la philosophie autrichienne et de sa place», in J.P. Cometti et K. Mulligan (dir.), La philosophie autrichienne de Bolzano à Musil. Histoire et actualité, Paris, Vrin, 2001, p. 825.

Reinach, Adolf. Sämtliche Werke, deux volumes, Munich, Philosophia, 1989.

Rojszczak, Artur et Barry Smith. "Truthmakers, Truthbearers and the Objectivity of Truth », in J. Hintikka, T. Czarnecki, K. Kijania-Placek, T. Placek, A. Rojszczak 
(dir.), Philosophy and Logic. In Search of the Polish Tradition, Dordrecht, Kluwer Academic Publishers, 2003, p. 229-268.

Srzednicki, Jan. Franz Brentano's Analysis of Truth, La Haye, Martinus Nijhoff, 1965.

Smith, Barry. Austrian Philosophy. The Legacy of Franz Brentano, Chicago, Open Court, 1994.

Stumpf, Karl. Tonpsychologie. Zweiter Band, Leipzig, Hirzel, 1890.

—. "Erscheinungen und psychische Funktionen", Abhandlungen der Königlich Preußischen Akademie der Wissenschaften. Philosophisch-historische Abhandlungen, vol. IV, 3-40, Berlin, Reimer, 1906.

- . "Phénomènes et fonctions psychiques", in Karl Stumpf, Renaissance de la philosophie. Quatre articles, introduction et traduction de D. Fisette, Paris, Vrin, 2006, p. 133-167 (trad. fr. de Stumpf, 1906).

—. "Autobiographie", in Karl Stumpf, Renaissance de la philosophie. Quatre articles, introduction et traduction de D. Fisette, Paris, Vrin, 2006a, p. 255307.

Tănăsescu, Ion. «'Der Vorstellungsgegenstand'. Zu Twardowskis Rezeption der Psychologie Brentanos ", in Brentano Studien, vol. 8, 1998, p. 53-66.

Twardowski, Kazimierz. 1894. Zur Lehre vom Inhalt und Gegenstand der Vorstellungen. Eine psychologische Untersuchung, Vienne, Hölder (réimpression Munich, Philosophia, 1982).

—_. "Funktionen und Gebilde», in Conceptus, vol. 29, n 75, 1996, p. 157-196.

- «Fonctions et formations. Quelques remarques aux confins de la psychologie, de la grammaire et de la logique ", in Denis Fisette et Guillaume Fréchette (dir.), À l'école de Brentano. De Würzburg à Vienne, Paris, Vrin, 2007, p. 343384 (trad. fr. de Twardowski [1996]).

Utitz, Emil. Die Funktionsfreude im ästhetischen Verhalten, Halle, Niemeyer, 1911. Werle, Josef M. Franz Brentano und die Zukunft der Philosophie. Studien zur Wissenschaftssystematik im 19. Jahrhundert, Amsterdam, Rodopi, 1989. 